Zdravko Kravanja (Editor), Proceedings of the $26^{\text {th }}$ European Symposium on Computer Aided Process Engineering - ESCAPE 26

June 12th -15th, 2016, Portorož, Slovenia (C) 2016 Elsevier B.V. All rights reserved.

\title{
Optimization and Monte Carlo Simulation for Product Launch Planning under Uncertainty
}

\author{
Catarina M. Marques ${ }^{\mathrm{a}}$, Samuel Moniz $\mathrm{z}^{\mathrm{b}}$, Jorge Pinho de Sousa ${ }^{\mathrm{a}, \mathrm{b}^{*}}$, Ana Paula \\ Barbosa-Póvoa $^{\mathrm{c}}$ \\ ${ }^{a}$ Faculdade de Engenharia da Universidade do Porto, Rua Dr. Roberto Frias, Porto, \\ Portugal \\ ${ }^{b}$ INESC TEC, Rua Dr. Roberto Frias, Porto, Portugal \\ ${ }^{c}$ Centro de Estudos de Gestão, Instituto Superior Técnico, Universidade de Lisboa, Av \\ Rovisco Pais, Lisboa, Portugal \\ jorge.p.sousa@inesctec.pt
}

\begin{abstract}
This paper presents an innovative approach to solve the product-launch planning problem in the pharmaceutical industry, with uncertainty on the product demand and on clinical trials. A mixed integer linear programming (MILP) model, incorporating Monte Carlo simulation (MCS), was developed for optimizing the process design (process-unit allocation and scale-up decisions) and for capacity planning (acquisition of new units), considering the products that still require development, and the products that are already in commercialization. MCS is performed in a two-step procedure, based on Normal and Bernoulli distributions, in order to capture the effects of demand variability and trials pass-fail uncertainty, respectively. Product-launch decisions are made taking into account the probability distributions of alternative process designs, of new capacity requirements, and of the coefficients of the objective function. The applicability of the proposed solution approach is demonstrated in an illustrative case study.
\end{abstract}

Keywords: Process Design, Capacity Planning, Pharmaceutical Industry, MILP, Monte Carlo Simulation.

\section{Introduction}

In the pharmaceutical industry, uncertainty is particularly relevant in product-launch decisions, as a high level of variability exists in the process and in the product demand. Time-to-market is a key issue in this industry and therefore, as the demand increases during the various phases of the product-launch process, all required resources need to be available in time to start production. Design and planning decisions, such as process to unit assignment, scale-up, and unit acquisition decisions, are then critical, and should be made early enough to guarantee the necessary availability of resources. However, such decisions are often made based on incomplete and inaccurate data, and therefore to integrate uncertainty into the planning models is of utmost importance. Model-based approaches can then be applied to effectively support the decision-making process of product-launch planning.

The main contribution of the approach proposed in this work is the integration of design and planning decisions. For this purpose, process design decisions take into account the resource limitations associated to the need of accommodating, simultaneously and in the same plant, the manufacture of products under development and products already in 
commercialization, with uncertainty concerning demand and clinical trials. Moniz et al. (2014) considered the production planning of multipurpose batch plants in which both types of products compete for the same resources, but they have not included uncertainty in the models. To simultaneously address uncertainties on product demand and clinical trials, this paper proposes a MILP model for optimal product-launch planning, combined with a two-step MCS framework that handles uncertainty with Normal and Bernoulli distributions. As a result, the proposed approach efficiently integrates the demand variability for both types of products, and the uncertainty on clinical trials for new products.

The remainder of the paper is structured as follows. Section 2 presents a brief literature review of the most common methods used for design and planning under uncertainty. Section 3 presents the problem description and its mathematical formulation. An example is presented in section 4 , and final remarks are made in section 5.

\section{Literature Review}

In process industry applications, and in particular in the pharmaceutical industry, a method commonly used for planning under uncertainty is two-stage Stochastic Programming (SP). Planning decisions are typically taken in two stages. Strategic decisions ("here and now") are made in the first stage, while operational decisions ("wait and see") are made in a second stage. Uncertainties are usually modelled as a set of discrete scenarios to account for all possible future outcomes.

Gatica et al. (2003) present a multistage SP formulation for capacity planning under uncertainty, in the pharmaceutical industry. This approach is used to select the final product portfolio, and simultaneously to perform production planning and to define the investment strategy, subject to the uncertainty on the outcomes of the clinical trials. More recently, Colvin and Maravelias (2008) also propose a scenario-based multi-stage SP model for planning the clinical trials. Sundaramoorthy et al. (2012) develop a framework for capacity planning in order to ensure the availability of enough resources for the foreseen demands of products, and they have developed a multi-scenario, multiperiod MILP formulation that takes into account uncertainty in the outcome of clinical trials. The main drawback of the scenario-based approaches is the significant increase of the model size with the increase of the number of scenarios.

An alternative to this approach is Monte Carlo simulation (MCS) used to generate random instances for each uncertainty parameter. Bassett et al. (1997) present a framework for including uncertainty parameters into a general aggregate production planning procedure, or resource constrained scheduling problems, using MCS. Farid et al. (2005) use MCS to model technical and market uncertainties based on a hierarchical framework. More recently, Eberle et al. (2014) present a framework for measuring and improving the production lead time of pharmaceutical processes, employing MCS, sensitivity analysis, and what-if analysis. When dealing with scenario-based approaches, not only formulation intractability is a downside, but also the determination of which scenarios to generate can be a complex problem. Particularly when dealing with multiple uncertainty parameters, the decision about what scenarios to generate is neither simple nor obvious. Additionally, the (sensitivity) analysis of each scenario tends to be very complex, and time consuming. To overcome these difficulties, the two-step MCS approach proposed in this work can be a sound alternative. It enables the investigation of a large number of possible values (instead of just scenarios), and a comprehensive analysis and assessment of the risks associated with each uncertainty parameter. 


\section{Proposed Method}

\subsection{Problem Definition}

Product development, as considered in this work, encompasses the clinical trials phases I, II, and III, and ends with the regulatory approval and the product launch. The main goal of the problem addressed here is to determine the optimal plan, including process to unit assignment, scale-up, and unit acquisition decisions, for the demand requirements of both the under development and the developed products. The problem can be defined as follows. We are given: $(i)$ the recipes of the products under development and already in commercialization; ( $i$ i ) the set of processing units available and their maximum and minimum capacities; (iii) the task suitability for every processing unit and task processing times; (iv) a fixed time planning horizon, discretized into equal duration intervals; $(v)$ the operational and investment costs associated to each task and unit available; and (vi) the probabilistic distributions of the demand and of the clinical trials pass-fail uncertainty. The objective is to maximize the overall profit, by determining: the size and timings of scale-up decisions; the best set of processing unit types for each process; and the capacity extension requirements.

\subsection{Solution Approach}

The solution approach developed in this work integrates a MILP model with MCS. The MCS component randomly generates a large number of instances, and for each of these instances, the MILP model is solved, and an optimal solution obtained. To model the uncertainty associated with the clinical trial outcomes, the probability of success of each product at the end of the trial phases is given by Bernoulli distributions, since there are only two possible results of the clinical tests: "success" or "failure". On the other hand, Normal distributions have been considered to capture the essential characteristics of demand uncertainty throughout each phase. Thus, in each iteration both uncertainty parameters (clinical trial outcomes, and product demand) are randomly sampled from their given probability distributions. This process is performed for each of the 3 clinical trial phases and for each product, in two main steps: $(i)$ random product demand generation; and (ii) random trial outcome generation. Figure 1 shows a schematic diagram of this procedure.

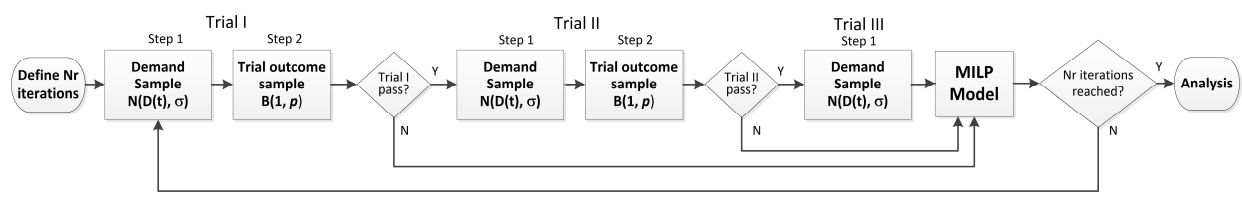

Figure 1 - Schematic representation of the two-step MCS

In short, the procedure works as follows. At the end of each trial phase, a sample trial outcome is performed in order to determine if the product successfully passed the clinical test. If so, a demand sample for the next clinical trial is generated. The sampled values are then used to run the MILP model, and the procedure is repeated until a certain number of iterations is reached. At the end, the probability density function for the objective function is determined and the results for the probabilistic occurrence of the decision variables are derived. These results are used to support decision-making concerning the design of the processes and the need for capacity extensions in each phase of the clinical tests. 


\subsection{MILP Model}

The formulation developed in this work is an aggregate planning model based on the RTN (Resource-Task Network) process representation, as introduced by Pantelides (1994). All material requirements as well as all storage levels and production yields are precisely defined through the model parameters and decision variables. However, the detailed time and task-sequencing constraints are not modeled. The optimal plan will be determined considering that production resources are shared by products under development and products already in commercialization. The formulation is defined by the following main constraints: $(i)$ resource balance constraints, to determine the materials availability over time (intermediates and final products); (ii) capacity constraints, to set the minimum and maximum boundaries for resources availability; (iii) demand constraints, to define the production requirements; (iv) batch size constraints, for ensuring that the total amount of material processed is within the capacities of the units; and $(v)$ plant capacity constraints, to express the time availability of the processing units, including the possibility to expand capacity. The objective function is the maximization of the profit, as defined by expression (1) (i.e. the total income over the time horizon, minus operational costs, investment costs (associated with the increase in capacity), storage costs, changeover costs, costs associated with the selection of processing units, and costs associated with missing deliveries).

$$
\begin{aligned}
\max \left[\sum_{m \in P} \sum_{t \in T}\left(\Pi_{m t} s_{m}\right)\right. & -\sum_{k \in K_{e}} \sum_{t \in T}\left(c_{k}^{\text {oper. }} \xi_{k, t}\right)-\sum_{e \in E} \sum_{t \in T}\left(c_{e}^{\text {invest. }} \delta_{e t}\right) \\
& \left.-\sum_{m \in M_{t}} \sum_{t \in T} c_{m}^{\text {stor. }} R_{m t}-\sum_{e \in E} \sum_{p \in P} \sum_{t \in T} c_{e}^{\text {chg. }} Y_{p e t}-\sum_{m \in P} \sum_{t \in T} \alpha_{p} \Pi_{m t}^{\text {slack }}\right]
\end{aligned}
$$

The key decision variables of the proposed model are: (i) selection of processing units binary variables $Y_{\text {pet }}\left(Y_{\text {pet }}=1\right.$ if product $p$ is assigned to equipment $e$, in time interval $t$ ); (ii) when and how much capacity to expand - integer variables $\delta_{e t}$; and (iii) total amount produced in each processing unit - continuous variables $\xi_{k t}$.

\section{Preliminary computational results}

To demonstrate the applicability of the proposed framework, the following product portfolio has been considered: 3 new products (PA, PB, and PC) entering in the development phase, and 2 products (PD and $\mathrm{PE}$ ) already in commercialization. The demand forecast profiles for each product are illustrated in Figure 2a). A 5 years planning horizon was considered, divided in equal time intervals of 6 months. The recipes for the products are given, including their yields, processing times, and alternative processing unit types. All processes are composed by 3 aggregate tasks that can be processed in 3 possible unit types $(\{\mathrm{R} 1, \mathrm{R} 2, \mathrm{R} 3\},\{\mathrm{F} 1, \mathrm{~F} 2, \mathrm{~F} 3\}$, and $\{\mathrm{D} 1, \mathrm{D} 2$, D3 $\}$ ) having different capacities and investment costs. These tasks have a variable duration that is proportional to the batch size.

\subsection{Results}

The MCS component was run for 1000 iterations, thus sampling the demand variability of the under development products. For this purpose, a normal distribution was considered, with values, per time period, for the mean and standard deviation of $30 \%$, derived from the data in Figure 2a). The clinical trial outcomes are determined by the Bernoulli distributions according to given success probabilities. For the products 
already in commercialization, a lower demand variability was considered (with a standard deviation of $10 \%$ ). The values of the objective function show that the profit follows a non-normal left-skewed probability distribution (see Figure 2b)).

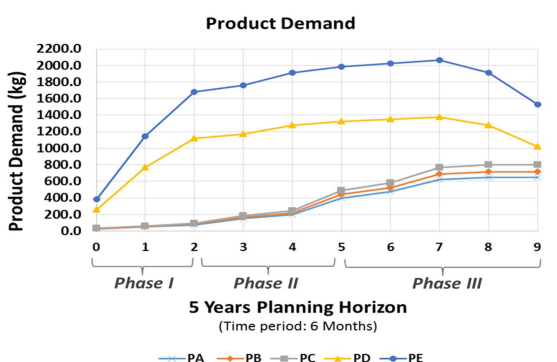

a)

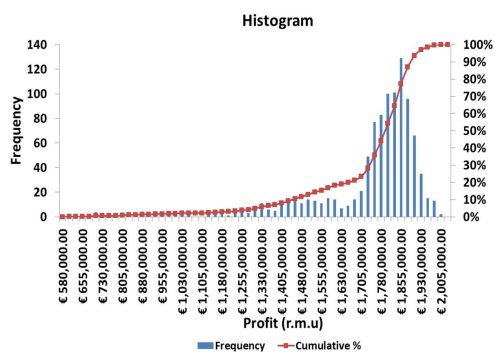

b)

Figure 2 - a) demand profile for products under development (PA, PB and PC) and products already in commercialization (PD and PE); and b) histogram and probability distribution of profit (objective function).

These results provide an interesting assessment of the impact of the product demand variability and clinical trial outcomes on the profit. The maximum profit value obtained was $2.00 \times 10^{6}$ relative monetary units, and the average profit was $1.73 \times 10^{6}$. Moreover, the most likely process design configurations for each product and clinical trial/planning period were determined. These results are illustrated in Figure 3.

Additionally, the most selected design configuration for products PD and PE includes the processing units with higher capacities for each task, not shown in the figure. Thus, unit R1 was selected for task 1, unit F1 for task 2 and unit D3 for task 3. This leads to larger and fewer batches for these products. The lower capacity units were seldom selected in this case. On the other hand, for the under development products, the most selected process design configurations are strongly connected to the success of the clinical trials (see Figure 3). So, the model favours the lower capacity units in clinical trials I and, along with the increase of the product demand for trials II and III, the model tends to use process design configurations with higher capacity units (whenever there is no capacity available in the smaller units). Clearly, the model seems to be handling well the trade-off between the allocation of the larger capacity units to the products with higher and stable demands (PD and PE) and the allocation of smaller capacity units to the products with higher demand variability (PA, PB, and PC).

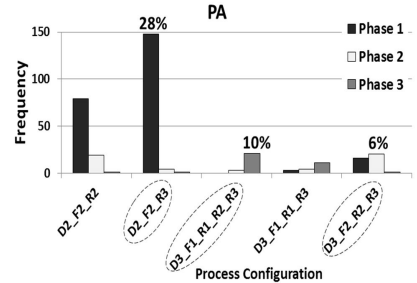

a)

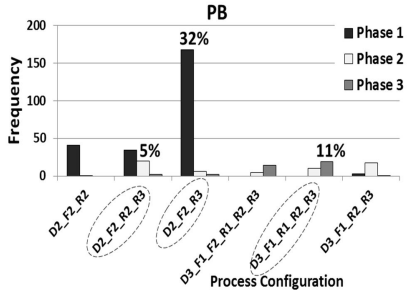

b)

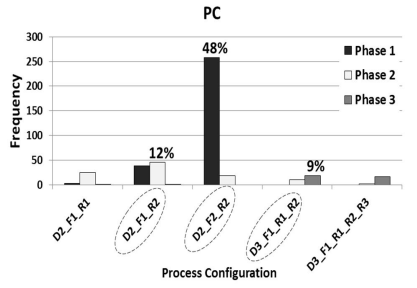

c)

Figure 3 - Probability of occurrence of each process design configuration for the products under development: a) product $\mathrm{PA}, \mathrm{b}$ ) product $\mathrm{PB}$, and c) product $\mathrm{PC}$.

Considering these results, the process design configurations D2_F2_R3 (Phase I), D3_F2_R2_R3 (phase II), and D3_F1_R1_R2_R3 (phase III) seem to be plausible 
choices to accommodate product PA. Similarly, for product PB the right choices appear to be the process configurations D2_F2_R3 (Phase I), D2_F2_R2_R3 (Phase II), and D3_F1_R1_R2_R3 (Phase III). Finally, for product PC the most frequently selected configurations were D2_F2_R2 (Phase I), D2_F1_R2 (Phase II), and D3_F1_R1_R2 (Phase III). In summary, we might say the solution approach proposed in this work provides the decision makers with valuable information regarding the process design configuration for profit maximization, taking into account the highly uncertain context involving product launch planning.

\section{Conclusion}

This paper presents an innovative solution approach (integrating optimization and Monte Carlo simulation) for the product launch planning problem, with uncertainty on the product demand and on clinical trials. This approach simultaneously considers the utilization of resources by products that are already in commercialization, and it can efficiently assess the effects of uncertainty in process design decisions (both processunit allocation and scale-up decisions) and in capacity planning, during product launch. Preliminary computational results indicate that the proposed approach provides a robust support to this decision-making process, through a clear identification of the most probable process configurations, in a highly uncertain context. Future research will address the systematization of the decision-making process, and the consideration of other relevant uncertainty parameters, such as production yield and lead times.

\section{Acknowledgement}

The authors gratefully acknowledge the financial support of Fundação para a Ciência e Tecnologia (FCT), under the grant PD/BD/105987/2014.

\section{References}

Bassett, M. H., Pekny, J. F., \& Reklaitis, G. V. (1997). Using detailed scheduling to obtain realistic operating policies for a batch processing facility. Industrial \& engineering chemistry research, 36, 1717-1726.

Colvin, M., \& Maravelias, C. T. (2008). A stochastic programming approach for clinical trial planning in new drug development. Computers \& chemical engineering, 32, 2626-2642.

Eberle, L. G., Sugiyama, H., \& Schmidt, R. (2014). Improving lead time of pharmaceutical production processes using Monte Carlo simulation. Computers \& chemical engineering, 68, 255-263.

Farid, S. S., Washbrook, J., \& Titchener-Hooker, N. J. (2005). Decision-Support Tool for Assessing Biomanufacturing Strategies under Uncertainty: Stainless Steel versus Disposable Equipment for Clinical Trial Material Preparation. Biotechnology progress, 21, 486-497.

Gatica, G., Papageorgiou, L., \& Shah, N. (2003). Capacity planning under uncertainty for the pharmaceutical industry. Chemical Engineering Research and Design, 81, 665-678.

Moniz, S., Barbosa-Póvoa, A. P., \& de Sousa, J. P. (2014). Simultaneous regular and non-regular production scheduling of multipurpose batch plants: A real chemical-pharmaceutical case study. Computers \& chemical engineering, 67, 83-102.

Pantelides, C. C. (1994). Unified Frameworks for Optimal Process Planning and Scheduling. In Proceedings on the Second Conference on Foundations of Computer Aided Operations (pp. 253-274).

Sundaramoorthy, A., Evans, J. M., \& Barton, P. I. (2012). Capacity planning under clinical trials uncertainty in continuous pharmaceutical manufacturing, 1: mathematical framework. Industrial \& engineering chemistry research, 51, 13692-13702. 